\title{
Higher Order Sliding Mode Observers in Power Grids with Traditional and Renewable Sources
}

\author{
Gianmario Rinaldi ${ }^{1}$, Prathyush P. Menon ${ }^{2}$, Christopher Edwards ${ }^{2}$ and Antonella Ferrara ${ }^{1}$
}

\begin{abstract}
This paper considers the application of higher order Sliding Mode (SM) observers to robustly and dynamically estimate the unmeasured state variables in modern power grids, in which both traditional and renewable energy sources coexist. In particular, a power grid composed of traditional, wind and inverter-based sources connected with dynamical loads is considered. Assuming that only the voltage phase angles are locally measured, a dedicated higher order SM observer is designed for each component, which is able to estimate in finite time the unmeasured state variables. Numerical simulations demonstrate the accuracy of the proposed scheme, also when compared with well-established linear observers.
\end{abstract}

Index Terms-Observers for nonlinear systems; Variablestructure/sliding-mode control; Power systems.

\section{INTRODUCTION}

$\mathbf{I}$ $\mathrm{N}$ recent years, a worldwide consensus has been reached to reduce greenhouse effects, by promoting the growth of renewable energy sources in power systems [1]. The installed capacity of these kinds of sources is increasing worldwide, especially led by wind power sources [2]. The incorporation of renewable sources in power systems can cause power mismatches between generation and demand, giving rise to frequency deviation and degradation of the power quality [3]. There is a shared and strong interest amongst researchers and practitioners for the design of more advanced and reliable strategies to model, control and monitor power systems with higher penetration of renewable sources [4], [5], [6].

Sliding Mode (SM) has been revealed to be an efficient technique for robust control and state estimation in complex dynamical systems, often affected by uncertainty conditions [7]. SM observation and control techniques have been applied in the power system context with the growing penetration of renewable energy sources. For example, in [8] a first order SM observer-based controller has been proposed to stabilise a traditional power plant. The effect of renewable sources has been accounted for in a lumped equivalent disturbance affecting the traditional power sources, and a disturbance observer has been created to estimate the lumped uncertainties. Differently, in [9] the wind power source was explicitly modelled, and an

1 Gianmario Rinaldi and Antonella Ferrara are with Department of Electrical, Computer and Biomedical Engineering, University of Pavia, Pavia, Italy. gianmario.rinaldioleuniversitadipavia.it, antonella.ferraraeunipv.it.

${ }^{2}$ Prathyush P. Menon and Christopher Edwards are with College of Engineering, Mathematics, and Physical Sciences, University of Exeter, Exeter, United Kingdom. P.M.Prathyush@exeter.ac.uk, C.Edwards@exeter.ac.uk.

This is the final version of the accepted paper included in the IEEE Control Systems Letters (L-CSS), 2019.
Unknown Input Luenberger (UIL) observer scheme coupled with a first order SM controller has been shown to stabilise the power network, in which the power flow was modelled via a DC (linearised) method.

Main Contribution: In this paper, higher order sliding mode observer schemes are developed to robustly estimate in finite time the states of a dynamical model of a comprehensive, hybrid power system constituting of traditional, wind and inverter based power sources and loads. In contrast to the SM observer schemes reported in the existing literature, in either traditional power grid models [10], [8], or renewable power sources [11], [12], which are independently considered, the present paper focuses on a model where heterogeneous power sources and loads are simultaneously present and are coupled through the power flow exchanges. As a consequence, the level of unknown, but bounded fluctuations and uncertainties that an observer would have to cope with, is significantly higher in this case than in the conventional independent cases [13]. Aligning to ongoing, novel wide area monitoring developments as reported in [13], only phase angles are assumed to be measured using Phasor Measurement Units (PMUs). Furthermore, it is assumed that the input signals, such as valve control and wind torque signals for governor and wind turbine sources respectively, are known and available for use in the observer design. Making use of the underlying structure of the dynamics of the coupled components in the power grid model, a fourth order sliding mode observer is designed for conventional and wind power sources, whereas a super-twisting like sliding mode observer structure as in [14] is used for load and inverter based power source components in the hybrid power system dynamic model. The proposed observers are shown to be totally insensitive to perturbations in power flow, which constitutes the couplings amongst the network components. Furthermore, a sensitivity analysis of the observation scheme is also undertaken. The proposed observation scheme is heterogeneous, since it consists of observers for different types of components. The proposed approach differs from the earlier works. For example in [10] a first order SM observer has been designed for hydrothermal power systems, and they have been able to asymptotically estimate the unmeasured state variables. In contrast, the proposed scheme aims to estimate the states in finite time. In contrast with [9], in which an UIL observer was developed, in the current framework the SM architecture is used, and its higher accuracy with respect to linear observers is demonstrated in the numerical examples. To the best of the authors' knowledge, the use of SM observers applied to hybrid power systems is novel, and it has never been developed before. 
Table I

LIST OF SyMBOLS AND VARIABLES USED IN THE MANUSCRIPT

\begin{tabular}{r|l} 
Symbol and Units & Meaning \\
\hline$\theta_{g}$ (rad) & traditional source voltage phase angle \\
$\theta_{w 2}$ (rad) & wind turbine angle and generator phase angle \\
$\theta_{s}, \theta_{l}$ (rad) & inverter source and load voltage phase angles \\
$\omega_{g}$ (p.u) & traditional source frequency \\
$\omega_{w 1}, \omega_{w 2}$ (p.u) & wind generator and turbine frequencies \\
$\omega_{s}, \omega_{l}$ (p.u) & inverter source and load frequencies \\
$P_{g}$ (p.u) & output turbine power \\
$v_{g}$ (p.u) & turbine valve position \\
$u_{g}$ (p.u) & valve control input \\
$T_{w}$ (p.u) & wind torque \\
$P_{s}$ (p.u) & inverter source input power \\
$P_{l}$ (p.u) & load power demand \\
$d_{e l}$ (p.u.) & power flow disturbances \\
$E_{g}$ (p.u) & traditional turbine self regulation \\
$k_{g}$ (p.u) & control valve position parameter \\
$r_{g}$ (p.u) & turbine speed droop \\
$T_{g 1}, T_{g 2}(\mathrm{~s})$ & turbine and servomotor time constants \\
$M_{g}$ (p.u) & traditional generator inertia \\
$M_{w 2}$ (p.u) & wind generator and wind turbine inertias \\
$M_{s}, M_{l}$ (p.u) & inverter (virtual) inertia and load inertia \\
$D_{g}, D_{w}$ (p.u) & traditional and wind source droop coefficients \\
$D_{s}, D_{l}$ (p.u) & inverter and load droop control coefficient \\
$k_{w}$ (p.u) & wind source stiffness coefficient \\
$\gamma_{i j}$ (p.u) & transmission line susceptance \\
$\omega^{\text {ref }}$ (p.u) & frequency reference value \\
\hline &
\end{tabular}

Structure of the Manuscript: The remaining parts of the manuscript are structured as follows. Section II describes the dynamical model for the considered power grid. Section III discusses key-assumptions and facts instrumental for the observers design. In Section IV dedicated higher order SM observers are proposed for each component of the grid, whilst in Section V the effectiveness of the proposed scheme is numerically demonstrated by simulations. Section VI concludes the paper.

Notation: The notation used throughout the manuscript is standard. For a given state variable $x, \hat{x}$ represents its estimate. The function $\operatorname{sign}(\cdot)$ is used for the signum function. The expression $\|\cdot\|$ represent the Euclidean norm. The expression $\operatorname{Col}(\cdot)$ defines a column vector. The symbol $x$ denotes a vector of appropriate dimensions with all its entries equal to $x$, where $x$ is a known scalar. Given the $i$-th component, the symbol $\mathcal{N}_{i}$ represents the indices of all the components directly adjacent to the $i$-th component. There can be multiple of these components, for example $n$ conventional generators, $m$ wind power source, $p$ inverters and $q$ loads. Table I shows the list of symbols and variables used throughout the paper, together with a brief description of their physical meanings.

\section{POWER GRID SySTEM Description}

In this section the dynamical model for the power grid will be briefly described. The considered power grid consists of an interconnection of four types of components: traditional power sources, wind power sources, inverter power sources, and loads. Each component interacts to its neighbourhood via power flow exchanges, which are allowed by transmission lines. Figure 1 shows a typical power grid in which the the four considered components are depicted. The numerical representations of the model parameters will be used later in the manuscript.

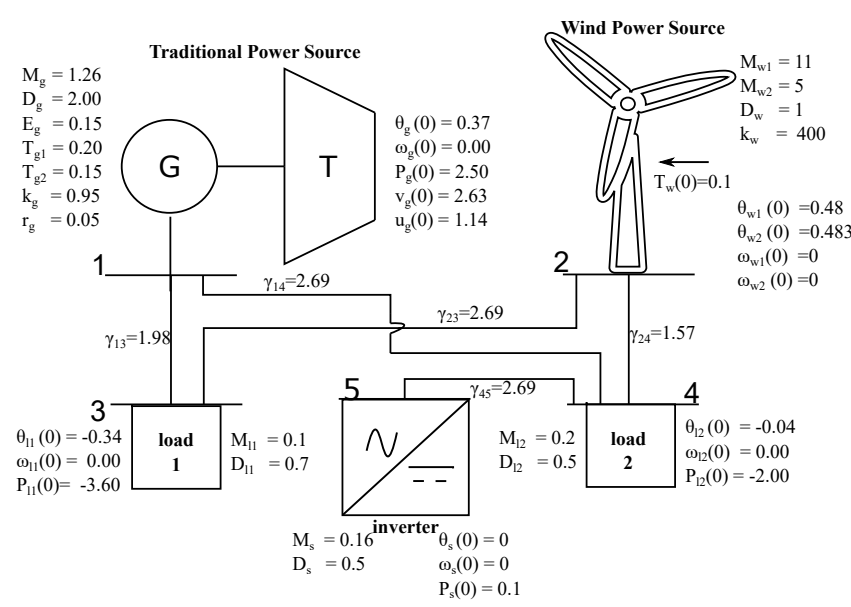

Figure 1. A schematic of the considered power grid (base power is set equal to 10 (MVA)). The numerical values for the model parameters and the initial conditions for the state variables will be used for the simulation assessment in Section V.

\section{A. Traditional Power Source Dynamical Model}

The traditional generation source typically consists of a turbine-governor unit which moves a synchronous generator [13]. Its dynamical model is described by [5], [13]:

$$
\begin{aligned}
\dot{x}_{g} & =A_{g} x_{g}+B_{g 1} u_{g}+B_{g 2}\left(P_{e g}\left(y_{g}, y_{\left.j\right|_{j \in \mathcal{N}_{g}}}\right)+d_{e g}\right) \\
y_{g} & =C_{g} x_{g}
\end{aligned}
$$

where

$$
\begin{aligned}
x_{g} \triangleq & {\left[\begin{array}{llll}
\theta_{g} & \omega_{g} & P_{g} & v_{g}
\end{array}\right]^{T} } \\
A_{g} \triangleq & {\left[\begin{array}{cccc}
0 & 1 & 0 & 0 \\
0 & \frac{E_{g}-D_{g}}{M_{g}} & \frac{1}{M_{g}} & 0 \\
0 & 0 & -\frac{1}{T_{g 1}} & \frac{k_{g}}{T_{g 1}} \\
0 & -\frac{1}{T_{g 2}} & 0 & -\frac{r_{g}}{T_{g 2}}
\end{array}\right] } \\
B_{g_{1}} \triangleq & {\left[\begin{array}{llll}
0 & 0 & 0 & \frac{1}{T_{g 2}}
\end{array}\right]^{T}, B_{g_{2}} \triangleq\left[\begin{array}{llll}
0 & -\frac{1}{M_{g}} & 0 & 0
\end{array}\right]^{T} } \\
C_{g} \triangleq & \triangleq\left[\begin{array}{llll}
1 & 0 & 0 & 0
\end{array}\right]
\end{aligned}
$$

In (1) the signal $P_{e g}\left(y_{g}, y_{\left.j\right|_{j \in \mathcal{N}_{g}}}\right)+d_{e g}$ denotes the total electrical active power flowing from the traditional power source to its neighbourhood $\mathcal{N}_{g}$, and it is defined as

$$
P_{e g}\left(y_{g}, y_{\left.j\right|_{j \in \mathcal{N}_{g}}}\right)+d_{e_{g}} \triangleq \sum_{j \in \mathcal{N}_{g}} \gamma_{g j} \sin \left(y_{g}-y_{j}\right)+d_{e g}
$$

The term $y_{j}$ brings further interactions from its neighbourhood $\mathcal{N}_{g}$, which could be any type from the heterogeneous components set.

\section{B. Wind Power Source Dynamical Model}

The so-called two-mass system is adopted in this work for dynamics of wind turbines, and it is described by [15]:

$$
\begin{aligned}
& \dot{x}_{w}=A_{w} x_{w}+B_{w 1} T_{w}+B_{w 2}\left(P_{e w}\left(y_{w}, y_{\left.j\right|_{j \in \mathcal{N}_{w}}}\right)+d_{e w}\right) \\
& y_{w}=C_{w} x_{w}
\end{aligned}
$$


where:

$$
\begin{aligned}
& x_{w} \triangleq {\left[\begin{array}{llll}
\theta_{w 1} & \omega_{w 1} & \theta_{w 2} & \omega_{w 2}
\end{array}\right]^{T} } \\
& A_{w} \triangleq {\left[\begin{array}{cccc}
0 & 1 & 0 & 0 \\
\frac{-k_{w}}{M_{w 1}} & -\frac{D_{w}}{M_{w 1}} & \frac{k_{w}}{M_{w 1}} & \frac{D_{w}}{M_{w 1}} \\
0 & 0 & 0 & 1 \\
\frac{k_{w}}{M_{w 2}} & \frac{D_{w}}{M_{w 2}} & -\frac{k_{w}}{M_{w 2}} & -\frac{D_{w}}{M_{w 2}}
\end{array}\right] } \\
& B_{w 1} \triangleq \triangleq\left[\begin{array}{lllll}
0 & -\frac{1}{M_{w 1}} & 0 & 0
\end{array}\right]^{T}, B_{w 2} \triangleq\left[\begin{array}{llll}
0 & 0 & 0 & \frac{1}{M_{w 2}}
\end{array}\right]^{T} \\
& C_{w} \triangleq\left[\begin{array}{llll}
1 & 0 & 0 & 0
\end{array}\right]
\end{aligned}
$$

In (3) the signal $P_{e w}\left(y_{w}, y_{\left.j\right|_{j \in \mathcal{N}_{w}}}\right)+d_{e w}$ denotes the total electrical active power flowing from the wind power source to its neighbourhood $\mathcal{N}_{w}$, and it is defined as:

$$
P_{e w}\left(y_{w}, y_{\left.j\right|_{j \in \mathcal{N}_{w}}}\right)+d_{e w} \triangleq \sum_{j \in \mathcal{N}_{w}} \gamma_{w j} \sin \left(y_{w}-y_{j}\right)+d_{e w} .
$$

\section{Inverter-Based Power Source Dynamical Model}

Inverters in renewable energy source applications can be controlled in such a way that they can mimic the behaviour of synchronous generators. In recent years, the so-called inverters with capacitive inertia [16] have been formulated, and they are described by

$$
\begin{aligned}
\dot{x}_{s} & =A_{s} x_{s}+B_{s} \phi_{s}\left(x_{s}, y_{\left.j\right|_{j \in \mathcal{N}_{s}}}, P_{s}, d_{e_{s}}\right) \\
y_{s} & =C_{s} x_{s}
\end{aligned}
$$

where the vectors and matrices are:

$$
x_{s} \triangleq\left[\begin{array}{c}
\theta_{s} \\
\omega_{s}
\end{array}\right], A_{s} \triangleq\left[\begin{array}{cc}
0 & 1 \\
0 & -\frac{D_{s}}{M_{s}}
\end{array}\right], B_{s} \triangleq\left[\begin{array}{l}
0 \\
1
\end{array}\right], C_{s} \triangleq\left[\begin{array}{ll}
1 & 0
\end{array}\right]
$$

In (5) the signal $\phi_{s}\left(x_{s}, y_{\left.j\right|_{j \in \mathcal{N}_{s}}}, P_{s}, d_{e s}\right)$ is given by

$$
\phi_{s}\left(x_{s}, y_{\left.j\right|_{j \in \mathcal{N}_{s}}}, P_{s}, d_{e s}\right) \triangleq \frac{P_{s}-\sum_{j \in \mathcal{N}_{s}} \gamma_{s j} \sin \left(y_{s}-y_{j}\right)+d_{e s}}{\omega_{s}+\omega^{\mathrm{ref}}}
$$

where $\mathcal{N}_{s}$ is the neighbourhood of the inverter source.

\section{Load Dynamical Model}

In the present work, a dynamical load is considered, and its model can be shown to be [13]

$$
\begin{aligned}
& \dot{x}_{l}=A_{l} x_{l}+B_{l} \phi_{l}\left(y_{l}, y_{j \mid j \in \mathcal{N}_{l}}, P_{l}, d_{e l}\right) \\
& y_{l}=C_{l} x_{l} \\
& x_{l} \triangleq\left[\begin{array}{c}
\theta_{l} \\
\omega_{l}
\end{array}\right], A_{l} \triangleq\left[\begin{array}{cc}
0 & 1 \\
0 & -\frac{D_{l}}{M_{l}}
\end{array}\right] \quad B_{l} \triangleq\left[\begin{array}{l}
0 \\
1
\end{array}\right], C_{l} \triangleq\left[\begin{array}{ll}
1 & 0
\end{array}\right]
\end{aligned}
$$

In (7) the signal $\phi_{l}\left(y_{l}, y_{\left.j\right|_{j \in \mathcal{N}_{l}}}, P_{l}, d_{e l}\right)$ is given by

$$
\phi_{l}\left(y_{l}, y_{\left.j\right|_{j \in \mathcal{N}_{l}}}, P_{l}\right) \triangleq P_{l}-\sum_{j \in \mathcal{N}_{l}} \gamma_{l j} \sin \left(y_{l}-y_{j}\right)+d_{e l}
$$

\section{Assumptions AND FACTS}

In this section, important properties of the power grid system described in Section II will be discussed, together with key-assumptions, which are instrumental for the observers design.

Proposition 1 Given the dynamics in (1), (3), (5), and (7), the associated pairs $\left(A_{g}, C_{g}\right),\left(A_{w}, C_{w}\right),\left(A_{s}, C_{s}\right)$, and $\left(A_{l}, C_{l}\right)$ are all observable.

The proposition can be proven by directly calculation of the determinant of the Observability matrices associated with the pairs $\left(A_{g}, C_{g}\right),\left(A_{w}, C_{w}\right),\left(A_{s}, C_{s}\right)$, and $\left(A_{l}, C_{l}\right)$, which are always full ranks. In what follows, these assumptions are imposed:

Assumption 1 It is assumed that:

(A1) The signals $P_{e g}$ in (1) and $P_{e w}$ in (3) are considered to be known, since they can be practically reconstructed by a local exchange of information about the measurements.

(A2) The signals $d_{e g}$ in (1), $d_{e w}$ in (3), $d_{e s}$ in (5), and $d_{e l}$ in (7) are bounded, which means that $\left|d_{e g}\right| \leq \Delta_{d_{e g}},\left|d_{e w}\right| \leq \Delta_{d_{e w}}$, $\left|d_{e s}\right| \leq \Delta_{d_{e s}},\left|d_{e l}\right| \leq \Delta_{d_{e l}}$, where $\Delta_{d_{e g}}, \Delta_{d_{e w}}, \Delta_{d_{e s}}$, and $\Delta_{d_{e l}}$ are known positive constants.

(A3) The signal $\phi_{s}$ in (5) and $\phi_{l}$ in (7) are unknown but bounded, which means that $\left|\phi_{s}\right|<\Delta_{\phi_{s}},\left|\phi_{l}\right|<\Delta_{\phi_{l}}$ where $\Delta_{\phi_{s}}$ and $\Delta_{\phi_{l}}$ are known positive constants.

Remark 1 Note that the value of the power flowing from a component to its neighbourhood is always bounded by the maximum power capacity of the power transmission lines [13], which is equal to the line susceptance $\gamma_{i j}$ (this is true in the most conservative situation, which occurs when $\left.\sin \left(\theta_{i}-\theta_{j}\right)=1\right)$. In practice, reasonable values for the bounds of the uncertain signals $d_{e g}, d_{e w}, d_{e s}$, and $d_{e l}$ in Assumption 1 can be obtained according to:

$$
\begin{aligned}
& \Delta_{d_{e g}} \geq \sum_{j \in \mathcal{N}_{g}} \gamma_{g j}, \Delta_{d_{e w}} \geq \sum_{j \in \mathcal{N}_{w}} \gamma_{w j} \\
& \Delta_{d_{e s}} \geq \sum_{j \in \mathcal{N}_{s}} \gamma_{s j}, \Delta_{d_{e l}} \geq \sum_{j \in \mathcal{N}_{l}} \gamma_{l j}
\end{aligned}
$$

Let $\Delta_{P_{s}}$ and $\Delta_{P_{l}}$ be the maximum value of the modulus of the signals $P_{s}$ in (5) and $P_{l}$ in (7), and let $\Delta_{\omega_{s}}$ represent the minimum value of $\omega_{s}+\omega^{\mathrm{ref}}>0$ in (6). Then, given (9), reasonable values of the bounds for $\Delta_{\phi_{s}}$ and $\Delta_{\phi_{l}}$ can be obtained as:

$$
\begin{aligned}
\Delta_{\phi_{s}} & \geq \frac{\Delta_{P_{s}}+\Delta_{\text {des }}}{\Delta_{\omega_{s}}} \\
\Delta_{\phi_{l}} & \geq \Delta_{P_{l}}+\Delta_{d_{e l}}
\end{aligned}
$$

Remark 2 Note that the degree of the unknown inputs $d_{\text {eg }}$ in (1) and $d_{e_{w}}$ in (3) with respect to the measured output $y_{g}$ and $y_{w}$ are equal to 2 in both the two systems. Given Proposition 1 , and following the theoretical developments in [17], the dynamical systems in (1) and in (3) are strongly detectable.

\section{OBSERVERS DESIGN}

In this section, dedicated SM-based observers are introduced to dynamically track the unmeasured state variable in the three types of generation nodes and in the load nodes. 


\section{A. Traditional and Wind Power Sources Observers}

For the purpose of designing the observers, it is possible to rewrite both (1) and (3), which display a similar structure, in the following general form:

$$
\begin{aligned}
\dot{x} & =A x+B_{1} u_{1}+B_{2}\left(G\left(y, y_{\left.j\right|_{j \in \mathcal{N}}}\right)+d\right) \\
y & =C x
\end{aligned}
$$

where $x$ represents the state vector, $A, B_{1}, B_{2}$, and $C$ are vectors and matrices of appropriate dimensions, $G\left(y, y_{\left.j\right|_{j \in \mathcal{N}}}\right)$ is a scalar known nonlinearity which models the power flow exchanges, $u_{1}$ is the known control input, and $d$ is a bounded unknown signal (i.e. $|d| \leq \Delta_{d}$, where $\Delta_{d}$ is a known constant) representing disturbances and unmodelled dynamics in the power flow channel. The following is a special case of the theoretical developments in [17] (in which higher order SM observers were designed for both stable and unstable, observable and detectable linear systems with unknown inputs):

Given the dynamics in (11), Proposition 1, and Remark 2, consider the following SM observer for both the traditional and wind power sources:

$$
\begin{aligned}
\dot{z} & =A z+B_{1} u_{1}+B_{2} G\left(y, y_{\left.j\right|_{j \in \mathcal{N}}}\right)-L(C z-y) \\
\hat{x} & =z-\hat{e} \\
\hat{e} & =P \bar{e} \\
\hat{y} & =C z
\end{aligned}
$$

where $z$ and $\hat{x}$ represent two estimates of $x, L \in \mathbb{R}^{4 \times 1}$ is a design vector, $P \in \mathbb{R}^{4 \times 4}$ is a matrix to be designed. The auxiliary signal $\bar{e}=\operatorname{Col}\left(\bar{e}_{1}, \ldots, \bar{e}_{4}\right)$ is governed by the following fourth order SM architecture:

$$
\begin{aligned}
& \dot{\bar{e}}_{1}=-k_{1}\left|\bar{e}_{1}-e_{1}\right|^{3 / 4} \operatorname{sign}\left(\bar{e}_{1}-e_{1}\right)+\bar{e}_{2} \\
& \dot{\bar{e}}_{2}=-k_{2}\left|\bar{e}_{1}-e_{1}\right|^{2 / 4} \operatorname{sign}\left(\bar{e}_{1}-e_{1}\right)+\bar{e}_{3} \\
& \dot{\bar{e}}_{3}=-k_{3}\left|\bar{e}_{1}-e_{1}\right|^{1 / 4} \operatorname{sign}\left(\bar{e}_{1}-e_{1}\right)+\bar{e}_{4} \\
& \dot{\bar{e}}_{4}=-k_{4} \operatorname{sign}\left(\bar{e}_{1}-e_{1}\right)
\end{aligned}
$$

where $e_{1} \triangleq \hat{y}-y$ is the output observation error, $\bar{e}_{1}$ denotes an estimate of $e_{1}$, whilst the auxiliaries signals $\bar{e}_{2}, \ldots, \bar{e}_{4}$, represent successive time derivatives of $\bar{e}_{1}$. If the design constants $k_{1}, k_{2}, k_{3}, k_{4}$ are tuned according to the standard rules for the Levant's differentiator [18], and the design vector $L$ is chosen such that $(A-L C)$ is Hurwitz, then it will be shown that the estimate $\hat{x}$ satisfies in finite time $\hat{x}=x$.

By subtracting (11) from (12), the error dynamics yield

$$
\dot{e}=(A-L C) e-B_{2} d
$$

where $e \triangleq z-x$. The vector $L$ is designed to ensure that the matrix $(A-L C)$ is Hurwitz, thus assigning arbitrarily chosen eigenvalues. Since the pair $(A, C)$ is observable as proven in Proposition 1, the pair $(A-L C, C)$ is also observable, with the associated Observability Matrix $\tilde{O}$. By making use of the matrix $\tilde{O}$, the following change of coordinates is considered: $\tilde{e}=\tilde{O} e$ with the associated error dynamics in the new coordinates: $\dot{\tilde{e}}=\tilde{A} \tilde{e}-\tilde{B}_{2} d$. Note that following the change of coordinates $\tilde{C}=C$, and the matrix $\tilde{A}$ has the following structure [19] [17]:

$$
\tilde{A}=\tilde{O}(A-L C) \tilde{O}^{-1}=\left[\begin{array}{cccc}
0 & 1 & 0 & 0 \\
0 & 0 & 1 & 0 \\
0 & 0 & 0 & 1 \\
\tilde{a}_{1} & \tilde{a}_{2} & \tilde{a}_{3} & \tilde{a}_{4}
\end{array}\right]
$$

where $\tilde{a}_{1}, \ldots, \tilde{a}_{4}$ are known constants. It is easy to verify that only the first component $\tilde{e}_{1}$ of the vector $\tilde{e}=\left[\tilde{e_{1}}, \ldots, \tilde{e_{4}}\right]^{T}$ is known, thus coinciding with the output observation error. Since the matrix $\tilde{A}$ is Hurwitz, and the unknown input $d$ is assumed bounded, the time evolution of $\tilde{e}$ remains bounded [19]. The problem of finding in finite time the successive time derivative of $e_{1}$, which are bounded, can be solved by means of SM Levant's differentiator [18], as argued in [17]. This technique is governed by (13), which corresponds to a third order SM Levant's differentiator implemented in the so-called non-recursive form. The underlying idea is to make use of the output observation error $e_{1}$ and create the fourth order dynamical system in (13). In [18] it has been shown that each estimate generated by the differentiator converge to the actual value in finite time. The tuning rules for the positive design constants $k_{1}, \ldots, k_{4}$ can be found, e.g., in [20] and [7]. It follows in finite time: $\bar{e}_{1}=\tilde{e}_{1}=e_{1}, \bar{e}_{2}=\tilde{e}_{2}, \bar{e}_{3}=\tilde{e}_{3}, \bar{e}_{4}=\tilde{e}_{4}$. After the linear change of coordinates $\hat{e}=P \bar{e}$, where $P=\tilde{O}^{-1}$, it is possible to retrieve actual values of the error $\hat{e}$ in the original coordinates reference in finite time. These are used to compensate in real time the estimate $z$ by introducing the following algebraic expression

$$
\hat{x}=z-\hat{e} \text {. }
$$

According to the algebraic relation in (16), the condition $\hat{x}=x$ holds in finite time.

\section{B. Observer for the Inverter Sources and Loads}

In this section a super-twisting-like SM observer is employed to estimate in finite time the frequency deviation both in inverter with capacitive inertia and in loads. Note that the dynamics in (5) and (7) share the same structure, which can be rewritten as

$$
\begin{aligned}
\dot{\theta} & =\omega \\
\dot{\omega} & =a \omega+\phi \\
y & =\theta
\end{aligned}
$$

where $\theta$ denotes the (inverter and load) voltage phase angle and the $\omega$ the (inverter and load) frequency deviation, respectively, and in (17) the model parameter $a=-D / M$ is the ratio between the droop control and the inertia. The bounded unknown signal $\phi$ represents either $\phi_{s}$ and $\phi_{l}$. It follows that $|\phi| \leq \Delta_{\phi}$, where $\Delta_{\phi}$ is a known constant.

Given the dynamics (17), and Assumption 1, consider the following super-twisting-like SM observer [14]:

$$
\begin{aligned}
\dot{\hat{\theta}}= & \hat{\omega}-\alpha_{1}\left|e_{\theta}\right|^{1 / 2} \operatorname{sign}\left(e_{\theta}\right)-a e_{\theta} \\
\dot{\hat{\omega}=} & -\alpha_{2} \operatorname{sign}\left(e_{\theta}\right)-a^{2} e_{\theta} \\
& +a \hat{\omega}-a \alpha_{1}\left|e_{\theta}\right|^{1 / 2} \operatorname{sign}\left(e_{\theta}\right)
\end{aligned}
$$

where $\hat{\theta}$ is the estimate of $\theta, \hat{\omega}$ is the estimate of $\omega$, the output observation error $e_{\theta} \triangleq \hat{\theta}-\theta$, and $\alpha_{1}, \alpha_{2}$ are positive 
Table II

Performance Metrics $\mathcal{E}$ For the SM AND PI ObSERVERs (SMO,

PIO). VALUES EXPRESSED IN (mp.u.) $=1 e-3(p . u$. $)$. SENSITIVITY

ANAlysis is PRovided in the Right COLUMN, IN WHICH $\Delta A=30 \% A$

\begin{tabular}{r|rr|rr} 
& & & \multicolumn{2}{|c}{ Sensitivity } \\
node & SMO & PIO & SMO & PIO \\
\hline 1 & 2.29 & 4.06 & 136.90 & 159.60 \\
2 & 0.12 & 156.67 & 64.18 & 9489.00 \\
3 & 9.64 & 51.83 & 9.67 & 51.83 \\
4 & 9.78 & 27.26 & 9.78 & 27.26 \\
5 & 0.56 & 9.73 & 0.57 & 9.73
\end{tabular}

design constants. The error dynamics, which is determined by subtracting (17) from (18), can be shown to be

$$
\begin{aligned}
\dot{e}_{\theta}= & e_{\omega}-\alpha_{1}\left|e_{\theta}\right|^{1 / 2} \operatorname{sign}\left(e_{\theta}\right)-a e_{\theta} \\
\dot{e}_{\omega}= & -\alpha_{2} \operatorname{sign}\left(e_{\theta}\right)-a^{2} e_{\theta}+a e_{\omega} \\
& -a \alpha_{1}\left|e_{\theta}\right|^{1 / 2} \operatorname{sign}\left(e_{\theta}\right)-\phi
\end{aligned}
$$

where $e_{\omega} \triangleq \hat{\omega}-\omega$. Consider the auxiliary error variable $\bar{e}_{\omega} \triangleq$ $e_{\omega}-a e_{\theta}$. As argued in [14], the error dynamics in (19) can be further simplified, and they take the form:

$$
\begin{aligned}
\dot{e}_{\theta} & =\bar{e}_{\omega}-\alpha_{1}\left|e_{\theta}\right|^{1 / 2} \operatorname{sign}\left(e_{\theta}\right) \\
\dot{\bar{e}}_{\omega} & =-\alpha_{2} \operatorname{sign}\left(e_{\theta}\right)-\phi
\end{aligned}
$$

The system in equation (20) is in the form of the super-twisting SM structure [7]. If the design constants satisfy

$$
\alpha_{1}=1.5 \sqrt{\Delta_{\phi}}, \alpha_{2}=1.1 \Delta_{\phi}
$$

it follows that follows that $e_{\theta}=e_{\omega}=\bar{e}_{\omega}=0$ in finite time [7]. Therefore, the conditions $\hat{\theta}=\theta$ and $\hat{\omega}=\omega$ hold in finite time.

Remark 3 Consider the matrix $A$ in (11) with unknown bounded uncertainty on the model parameters as follows $A \pm$ $\Delta A$, where $\Delta A$ represents the maximum parameter deviations. The model uncertainty affects the observer error dynamics (14) as $\dot{e}=A e \mp(\Delta A) x-B_{2} d$. However, the time evolution of $e$ remains ultimate bounded as long as $x$ remains bounded. The compensation strategy in (16) is also affected. In particular, it can be shown that $\hat{x}=x \pm \Delta \hat{x}$, where the deviation $\Delta \hat{x}$ remains bounded. Furthermore, in case the model parameter a in (17) is uncertain, the super-twisting-like observer in (18) can be replaced via a standard super-twisting one [14]. It follows that in such a situation the impact of the model uncertainties can be totally circumvented.

\section{Simulation Results}

In this section, the effectiveness of the proposed SM observer scheme will be demonstrated by numerical simulations implemented in the Matlab-Simulink R2018a environment. The solver Odel (Euler Method) is employed for the numerical implementation of the observers, with a fixed integration step size equal to 10 microseconds. The adopted value of the sampling time is acceptable in practice, according to the technical data about the PMUs which are available in [21].

A power grid comprising a conventional power source, a wind power source, an inverter-based source, and two loads is considered. The base power for the network is chosen equal to 10 (MVA). The schematic in Figure 1 shows the system topology, together with the numerical representation of the model parameters and the initial conditions for the state variables and inputs. The data was take from [5] and [13]. The grid is considered to be at steady state during the time interval $0 \leq t \leq 5$ seconds. At the time instant $t=5$ seconds, the power generated by the inverter power source goes to zero, causing a loss of generation of 0.1 (p.u.). It follows that a disturbance in the form of $d_{e s}=-0.1 \operatorname{step}(t-5)$ appears in (6). Furthermore, a differentiable band-limited noise affects the measurements of all the voltage phase angles. These are simulated in Simulink choosing standard deviation equal to 3.00 (mrad).

The design constants for the observers in (12) for both the traditional and wind power sources are set as $k_{1}=25.5$, $k_{2}=72, k_{3}=220, k_{4}=330$, exploiting the tuning rules in [7]. By choosing $\Delta_{\phi}=300$, the designs constants of the observers in (21) are $\alpha_{1}=26, \alpha_{2}=330$. The proposed schemes in (12) and in (18) does not satisfy the rank conditions needed to use UIL [22], and hence are compared with PI observers, originally proposed in [23]. Using standard poleplacement techniques, the vector $L=\underline{100}, L \in \mathbb{R}^{4}$, is chosen for conventional and wind sources, whilst $L=\underline{100}, L \in \mathbb{R}^{2}$ is chosen for inverter sources and loads. According to Figure 2 , it is possible to demonstrate that the proposed SM scheme is able to faithfully estimate the unmeasured state variables, both when the grid is at steady-state and during the transient caused by the loss of generation from the inverter. In addition, the proposed observer is revealed to be totally insensitive to disturbances appearing in the matched channels of the systems (19). All the PI observers display less accuracy. Moreover, small oscillations can be noted in the estimates produced by the SM observers. These are due to the effect of the introduced measurement noise. The following performance metric can be introduced for all the observers in the noise-free scenario:

$$
\mathcal{E}=\frac{\int_{T_{1}=1}^{T_{f}=10}\|e(\tau)\| d \tau}{T_{f}-T_{1}}
$$

where the state estimation error vector $e$ differs for each considered observer. The interval of integration starts at the time instant $T_{1}=1$ seconds, when all the proposed observers have reached the sliding motion, and it stops at the time instant $T_{f}=10$ seconds, when the simulation ends. Table II demonstrates that the SM observers display smaller values w.r.t. the proposed metric. A sensitivity analysis is shown in Table II , where the matrix $A$ in (11) and the constant $a$ in (17) are affect by uncertainty of $30 \%$. In accordance with Remark 3, the observer (18) is totally insensitive to the model uncertainty, whilst the observer (12) is affected by this uncertainty, without compromising its stability. However, the SM observers always display a smaller value of the performance metric when compared to PI observers.

\section{CONCLUSIONS}

In this paper a comprehensive SM-based scheme has been developed for dynamic state estimation in power systems comprising both traditional and renewable power sources. This 

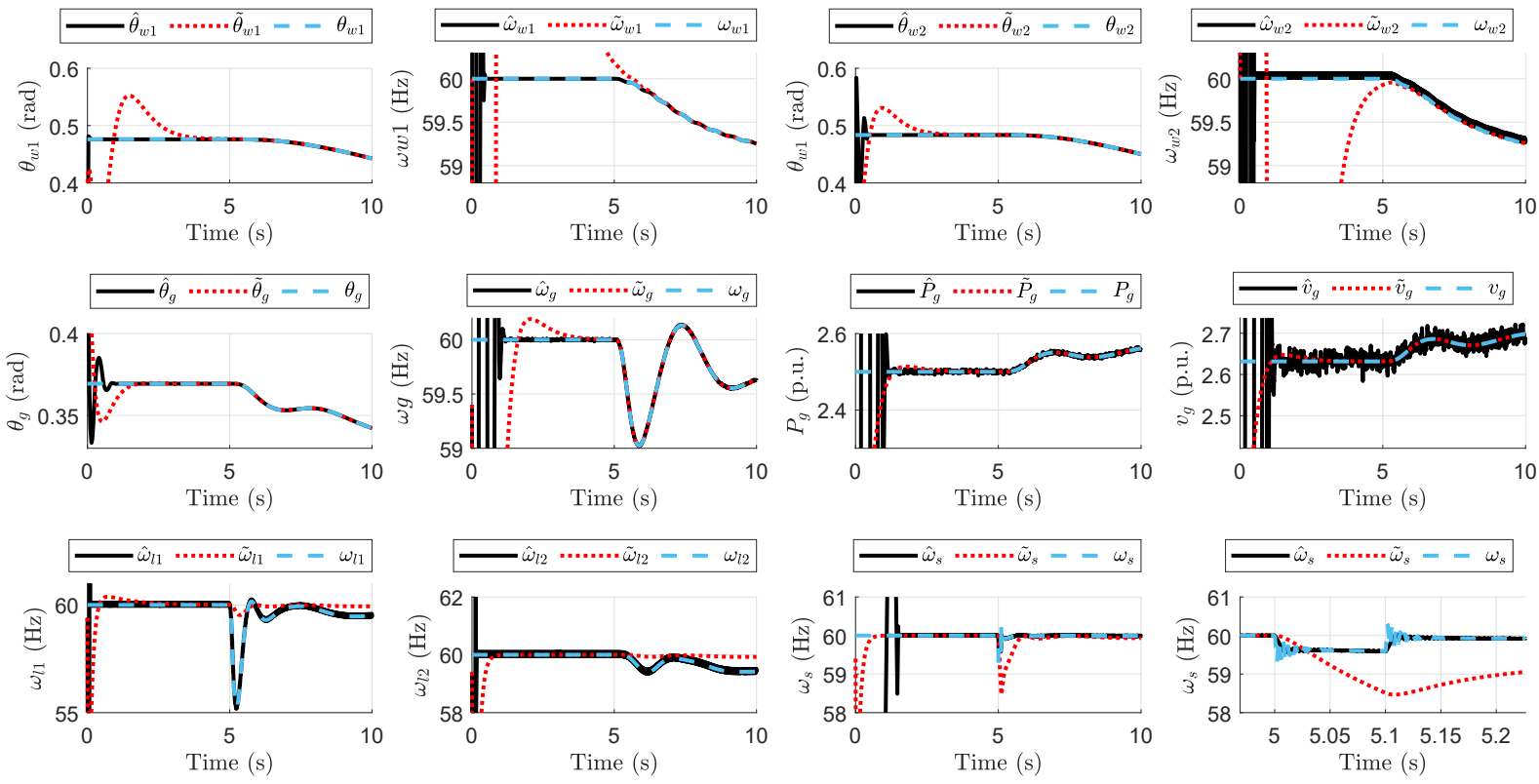

Figure 2. Time evolutions of: (a) $\theta_{g}, \omega g, P_{g}, v_{g}$, together with their estimates $\hat{\theta}_{g}, \hat{\omega}_{g}, \hat{P}_{g}, \hat{v}_{g}$, via proposed SM observer, and the estimates $\tilde{\theta}_{g}, \tilde{\omega}_{g}, \tilde{P}_{g}, \tilde{v}_{g}$ via PI observer. (b) $\theta_{w 1}, \omega w 1, \theta_{w 2}, \omega_{w 2}$, together with their estimates $\hat{\theta}_{w 1}, \hat{\omega}_{w 1}, \hat{\theta}_{w 2}, \hat{\omega}_{w 2}$, via proposed SM observer, and the estimates $\tilde{\theta}_{w 1}, \tilde{\omega}_{w 1}, \tilde{\theta}_{w 2}, \tilde{\omega}_{w 2}$ via PI observer. (c) $\omega_{l 1}, \omega_{l 2}$ and of inverter state variable $\omega_{s}$, together with their estimates $\hat{\omega}_{l 1}, \hat{\omega}_{l 2}, \hat{\omega}_{s}$, via proposed SM observer, and the estimates $\tilde{\omega}_{l 1}, \tilde{\omega}_{l 2}, \tilde{\omega}_{s}$ via PI observer. An enlarged view is provided for the inverter state variable and estimates.

paper distinguished from the earlier works in the literature, in which SM state observer was separately developed for traditional and renewable components in power grids. By means of higher order SM architecture it has been possible to enforce a finite time convergence of the observer error dynamics, thus ensuring better performances when compared to well-established linear observers.

\section{REFERENCES}

[1] T. Kåberger, "Progress of renewable electricity replacing fossil fuels," Global Energy Interconnection, vol. 1, no. 1, pp. 48-52, 2018.

[2] G. Boyle, Renewable energy. Oxford University Press, 2004.

[3] A. Ulbig, T. S. Borsche, and G. Andersson, "Impact of low rotational inertia on power system stability and operation," IFAC Proceedings Volumes, vol. 47, no. 3, pp. 7290-7297, 2014.

[4] S. Trip, M. Cucuzzella, C. De Persis, A. Ferrara, and J. M. Scherpen, "Robust load frequency control of nonlinear power networks," International Journal of Control, pp. 1-14, 2018.

[5] A. K. Bejestani, A. Annaswamy, and T. Samad, "A hierarchical transactive control architecture for renewables integration in smart grids: Analytical modeling and stability," IEEE Transactions on Smart Grid, vol. 5, no. 4, pp. 2054-2065, 2014.

[6] M. Cucuzzella, S. Trip, C. De Persis, X. Cheng, A. Ferrara, and A. van der Schaft, "A robust consensus algorithm for current sharing and voltage regulation in dc microgrids," IEEE Transactions on Control Systems Technology, no. 99, pp. 1-13, 2018.

[7] Y. Shtessel, C. Edwards, L. Fridman, and A. Levant, Sliding mode control and observation. Springer, 2014.

[8] A. S. Tummala, R. Inapakurthi, and P. Ramanarao, "Observer based sliding mode frequency control for multi-machine power systems with high renewable energy," Journal of Modern Power Systems and Clean Energy, vol. 6, no. 3, pp. 473-481, 2018.

[9] Y. Cui, L. Xu, M. Fei, and Y. Shen, "Observer based robust integral sliding mode load frequency control for wind power systems," Control Engineering Practice, vol. 65, pp. 1-10, 2017.

[10] G. Rinaldi, M. Cucuzzella, and A. Ferrara, "Sliding mode observers for a network of thermal and hydroelectric power plants," Automatica, vol. 98, pp. 51-57, 2018.
[11] B. Yang, T. Yu, H. Shu, J. Dong, and L. Jiang, "Robust sliding-mode control of wind energy conversion systems for optimal power extraction via nonlinear perturbation observers," Applied Energy, vol. 210, pp. 711723, 2018.

[12] I.-S. Kim, M.-B. Kim, and M.-J. Youn, "New maximum power point tracker using sliding-mode observer for estimation of solar array current in the grid-connected photovoltaic system," IEEE Transactions on Industrial Electronics, vol. 53, no. 4, pp. 1027-1035, 2006.

[13] P. W. Sauer, M. A. Pai, and J. H. Chow, Power system dynamics and stability: with synchrophasor measurement and power system toolbox. John Wiley \& Sons, 2017.

[14] G. Rinaldi, P. P. Menon, C. Edwards, and A. Ferrara, "Design and validation of a distributed observer-based estimation scheme for power grids," IEEE Transactions on Control Systems Technology, 2018.

[15] M. Singh and S. Santoso, "Dynamic models for wind turbines and wind power plants," National Renewable Energy Lab.(NREL), Golden, CO (United States), Tech. Rep., 2011.

[16] P. Monshizadeh, C. De Persis, T. Stegink, N. Monshizadeh, and A. van der Schaft, "Stability and frequency regulation of inverters with capacitive inertia," in Proc. of 2017 IEEE 56th Annual Conference on Decision and and Control (CDC), Melbourne, Australia, December 2017, pp. 5696-5701.

[17] L. Fridman, A. Levant, and J. Davila, "Observation of linear systems with unknown inputs via high-order sliding-modes," International Journal of Systems Science, vol. 38, no. 10, pp. 773-791, 2007.

[18] A. Levant, "Robust exact differentiation via sliding mode technique," automatica, vol. 34, no. 3, pp. 379-384, 1998.

[19] T. Kailath, Linear systems. Prentice-Hall Englewood Cliffs, NJ, 1980.

[20] E. Cruz-Zavala and J. A. Moreno, "Levant's arbitrary order exact differentiator: A lyapunov approach," IEEE Transactions on Automatic Control, 2018.

[21] Siemens, "SICAM, power quality and measurement," siemens.com/ powerquality, Tech. Rep., 2019, accessed: June 2019.

[22] C. Edwards and C. P. Tan, "A comparison of sliding mode and unknown input observers for fault reconstruction," European Journal of control, vol. 12 , no. 3, pp. 245-260, 2006.

[23] D. Söffker, T.-J. Yu, and P. C. Müller, "State estimation of dynamical systems with nonlinearities by using proportional-integral observer," International Journal of Systems Science, vol. 26, no. 9, pp. 1571-1582, 1995. 